livraisons

d'Histoire

de l'Architecture

\section{Livraisons de l'histoire de l'architecture}

$30 \mid 2015$

Le dessin d'architecture : œuvre/outil des architectes

?

\title{
La machine optique de l'architecture : desseins et dessins de Jean-Paul Jungmann
}

The optical engine in architecture: Jean-Paul Jungmann's drawings and intentions

Die optische Maschine der Architektur: Planungen und Zeichnungen von JeanPaul Jungmann

\section{Agnès Callu}

\section{OpenEdition \\ Journals}

Édition électronique

URL : http://journals.openedition.org/lha/549

DOI : $10.4000 /$ /ha. 549

ISSN : 1960-5994

Éditeur

Association Livraisons d'histoire de l'architecture - LHA

Édition imprimée

Date de publication : 30 décembre 2015

Pagination : 69-80

ISSN : 1627-4970

Référence électronique

Agnès Callu, «La machine optique de l'architecture : desseins et dessins de Jean-Paul Jungmann », Livraisons de l'histoire de l'architecture [En ligne], 30 | 2015, mis en ligne le 18 décembre 2017, consulté le 10 juin 2020. URL : http://journals.openedition.org/lha/549 ; DOI : https://doi.org/10.4000//ha.549 
Par Agnès Callu

\section{LA MACHINE OPTIQUE DE L'ARCHITECTURE : DESSEINS ET DESSINS DE JEAN-PAUL JUNGMANN}

Paul Valéry «il se peut que le dessin soit la plus obsédante tentation de l'esprit"

Évoquer les dessins de Jean-Paul Jungmann ouvre une rêverie esthétique, graphique et poétique enivrante, assurément, dense et attendant l'étude, plus encore. De telle sorte qu'observer la sûreté de son trait et les vagues d'une utopie datée «70 $»^{1}$ retient car, par-delà l'histoire d'une trajectoire individuelle, c'est bien une génération au travail, des projets in progress et une culture urbaine qui deviennent saillants et, dès lors, connaissent une inscription lourde dans le territoire de l'historien. À la hauteur d'une seule contribution, l'exercice panoramique n'est pas favorable quand il faut croiser biographie et stances de vie créatives. Pourtant, lui seul autorise l'interprétation, en épaisseur, de la place du dessin dans la construction «monumentée " d'une vie, d'une envie d'architecte. Aussi bien, une démonstration historique en trois mouvements chrono-thématiques est-elle à prévoir. D'abord, la montée en charge d'une construction identitaire ouverte aux influences telluriques de Mai 68. Après, au centre, une installation graphique dans la seconde moitié des années 1970 et jusqu'au coude des Eighties, qualifiable dans l'oxymore architecturale Utopie ${ }^{2}$ versus Réalisme qui entend Roland Barthes écrire que «l'architecture est toujours rêve et fonction " $(s i c)^{3}$. Enfin, un focus sur les détails d'un projet, en l'espèce ici, la Colline de Chaillot, pour partager, depuis l'intérieur, à partir du premier geste dessiné, la mise en place d'un processus créatif sans équivalent.

Le dossier critique de l'analyse est sûr car homogène. Le répertoire iconique est fourni par les dessins de J.-P. Jungmann rassemblés dans la collection graphique du musée des Arts décoratifs ${ }^{4}$. Les outils théoriques reposent, d'un côté, sur l'écriture, dense, de J.-P. Jungmann : ses auto-discours, ses notes de travail, ses conférences;

1. Jean-Paul Jungmann, "Voyage aux alentours d'Utopie », dans L'Architecture d'aujourd'hui, n 135 , décembre 1967, LIX-LXVI.

2. Norbert Elias, L'utopie [traduit de l'allemand par Hélène Leclerc et de l'anglais par Delphine Moraldo et Marianne Woollven ; introduction et supervision des traductions par Quentin Deluermoz], Paris, La Découverte, 2014.

3. Hans Ulrich Obrist, "À propos d'utopie et d'architecture : interview de Jean-Paul Jungmann ", 17 décembre 2001, [non pag.].

4. Musée des Arts décoratifs, département des Arts graphiques, corpus J.-P. Jungmann, cf. 2010.85.1.37 et FNAC-1870.1.38. 
de l'autre, sur la narration effectuée par ceux de son "réseau " ou celle que ses contemporains livrent sur lui et son travail. Enfin, à la manière d'un marqueur historiographique, il est impératif de considérer que l'étude se présente comme une recherche présentiste, donc issue de l'histoire du temps présent, écrite sous l'œil du témoin, avec lui, contre lui, près de lui encore, avec les anamorphoses produites par des séries d'objectivations et, dans le même instant, les subjectivités attachées à ce type d'exposé 5 .

À toutes forces et afin que l'invalidité de l'exemple unique soit immédiatement rejetée, inscrire J.-P. Jungmann dans sa classe d'âge est une nécessité. Né en 1935, il participe de la génération qui a connu les traumas de la Guerre, l'Algérie et "se retrouve" trentenaire en Mai 68. Dans l'enquête publiée en 2010 aux Presses du Septentrion sous le titre Le Mai 68 des historiens : entre histoire orale et identités narratives, sont expliquées les imprégnations socio-culturelles d'une telle classe d'âge ${ }^{6}$. Ainsi, c'est l'évidence, le facteur générationnel est déterminant quand il configure en épaisseur les aspirations d'un groupe, dès lors socialement mais intuitivement aussi, fédéré dans ses croyances par un/des événement(s) fondateur(s), des valeurs partagées, des figures fonctionnelles etc.

De la sorte, hors de son profil, à l'écart presque de ses goûts, de ses fondations et de ses références, J.-P. Jungmann est issu d'une classe emblématique : la " 35 " (sic), celle qui précède les Baby-boomers de la Libération. C'est pourquoi le fait générationnel se pose en note séminale puisqu'il est prescripteur pour analyser, en profondeur, l'influence matricielle des environnements, les postures sociétales, les curiosités, les prises de position d'un sujet "pensant et agissant".

Il faut traverser Strasbourg, la sphère familiale et la marée des envies esthétiques de J.-P. Jungmann pour arriver, rapidement, au seuil des années 68. Là, l'historiographie récente a rappelé l'implication décisive de l'architecte dans le "moment gonflable" (sic), en attelage avec Antonio Stinco et Jean Aubert ${ }^{7}$. On connait leur appui théorique: Frei Otto, créateur du Pavillon allemand de $\mathrm{Montréal}^{8}$. On se souvient qu'ils ont présenté leur projet de "Gonflables " pour le diplôme d'architecte aux Beaux-Arts en juillet 1967. Là, J.-P. Jungmann s'était centré, singulièrement, sur le petit volume évoquant le bonhomme Michelin : un assemblage de matelas pneumatiques et de ballons de football baptisé "Diodon ", du nom d'un poisson des mers qui enfle démesurément quand on le sort de l'eau.

Le postulat des trois jeunes architectes retient parce que, là encore, il est totémique d'une génération. Il faut bien en entendre le ressort. Ni Stinco, ni Aubert ni J.-P. Jungmann ne veulent ériger un nouveau système. Cela est même le contraire quand ils s'élèvent contre toutes les formes de systématisation qui veulent figer

5. Henry Rousso, La Dernière catastrophe : l'histoire, le présent, le contemporain, Paris, Gallimard, 2012.

6. Agnès Callu (dir.), Le Mai 68 des historiens : entre identités narratives et histoire orale [avant-propos de Michel Zink, préface de Jacques Revel et postface de Daniel Roche], Villeneuve-d'Ascq, Presses Universitaires du Septentrion, 2010.

7. Juliette Courtillier, "Les structures gonflables des années 1960 ", Serge Lemoine dir., mémoire de DEA, Paris IV, 2005.

8. Winfried Nerdinger, Frei Otto : complete works, Hardcover, mai 2005. 
l'architecture, celle du gonflable, du cube, de la sphère, de l'oblique et sq. Ils aspirent à défricher des voies nouvelles, à étudier des matériaux modernes qui autorisent la création de structures légères appliquées à des constructions mobiles. Leurs motsclefs ? Légèreté. Mobilité 9 . Les problèmes d'urbanisation, de circulation les attrapent de manière frontale. J.-P. Jungmann veut des habitations futuristes et elles sont à leur place dans les chantiers provisoires, au service d'expéditions polaires ou sahariennes (voire lunaires), en faveur d'hébergements en cas de catastrophes. Et cela fonctionne aussi pour l'aménagement intérieur car, avec l'ameublement pneumatique, le problème de place disparaît : dégonflés et pliés, les différents éléments n'occupent pas plus de volume qu'une pile de draps ${ }^{10}$.

En définitive, l'idée de confort - très "soixhante-huitarde " - est rectrice mais elle n'est pas univoque. De fait, en substrat, les A.J.S. (Aubert/Jungmann/Stinco) qui fondent en 1969 le groupe Aérolande, traversé de connaissances esthétiques, techniques, historiques et de voyages d'études, intègrent, précisément, le gonflable dans certains programmes d'architecture ${ }^{11}$. Le groupe défend la position suivante : de matériaux nouveaux naissent des formes nouvelles ${ }^{12}$. Mort du cube, bien sûr, mais aussi jeu de l'opaque, du transparent. En un mot, et dans la simplification, le gonflable provoque une sorte de happening permanent dans la mesure où, modifiant l'ensemble des paramètres d'une appréhension spatiale, il crée un univers ludique, rassurant, presque "un effet matriciel ». Il est immédiatement un objet architectural ; il qualifie " en live" l'espace; il accomplit l'architecture; il gomme le processus de construction, les savoir-faire, les techniques; il est l'objet préfabriqué par excellence : il transcende l'idéal du monde industriel, architecture de consommation au même moment qu'architecture jetable ${ }^{13}$.

On se rappelle aussi que de 1966 à 1971, les A.J.S. participent à la revue Utopie, revue de sociologie de l'urbain ${ }^{14}$ qui, promouvant démarches, débats et savoirs pluritransdisciplinaires, regroupe des chercheurs attachés à l'étude de la problématique urbaine $^{15}$. Là, la personnalité d'Hubert Tonka fonctionne à la manière d'un accélé-

9. Jean Aubert, Gérard Dietrich-Sainsaulieu, Jean-Paul Jugmann, Antoine Stinco, «Précisions sur la mobilité en architecture", dans Profil, nº 1 [Dossier l'Architecture magique], juillet-août 1975, [non pag.].

10. Voir, en particulier, contemporains, "Structures gonflables ", L'Humanité Dimanche, n 160, mars 1968 ; "Formes et techniques : le monde dynamique des structures gonflables ", Le Monde, 14 mars 1968 ; Claire de Bartillat, "L'air pour l'art... ou le domaine illimité des structures gonflables ", Combat, 3 avril 1968.

11. «A.J.S. Aérolande. Le Dyodon-Habitation 67 », Paris, Centre Pompidou, 2008.

12. Jean-Paul Jungmann, "L'ivresse du projet théorique", The inflatable moment: Pneumatics and protest in 68, New York, Architectural League of New York, 28 mai 1998.

13. Mobi boom : l'explosion du design en France, 1945-1975, Paris, Les Arts décoratifs, 2010.

14. Craig Buckley, "Interview with Jean-Paul Jungmann, co-éditor of Utopie: Sociologie de l'urbain from 1967-1969 (1970)", Paris, juin 2007.

15. Les thématiques soulevées dans Utopie, publiée aux éditions Anthropos, entre 1967 et 1969, sont éclairantes. À signaler : "Notes sur marxisme et esthétique "; "Devenir suranné "; "La répression »; "Villes de papier ", "Qui ne considèrera pas seulement le terrain strict du papier ", "Le rapport ville-nature dans les réalisations urbaines françaises de 1945 à 1965 ", "La logique de l'urbanisme ", novembre 1967 ; « Des raisons de l'architecture », mars-septembre 1968 ; « L'argent de l'urbanisme », 
rateur de particules. "Outsider curieux de tout ", dit de lui J.-P. Jungmann, il suit les séminaires de Foucault, Deleuze, est à Nanterre avec Henri Lefebvre, fréquente le "séminaire Tony Garnier " ${ }^{16}$, débat avec Jean Baudrillard "grand regardeur des choses" $(\text { sic })^{17}$.

Pour les A.J.S., l'architecte solitaire, entouré des formes nouvelles de la création, est une posture qui n'existe plus. Ils promeuvent le collectif et se montrent attentifs à une connaissance minutieuse de la société, de ses modes de production, des possibilités de l'industrie en ses matériaux nouveaux. Ils reconnaissent, admettent, acculturent l'impact des formes nouvelles sur l'utilisateur. Leur architecture n'est pas une esthétique qui arpenterait les marges de la Société. Ailleurs et plus loin, elle formalise un "nouveau monde", des rapports sociaux restructurés, des échanges entre l'homme et l'objet repensés. Loin de mettre en forme une société qu'ils critiquent, ils espèrent la questionner par l'architecture. L'enjeu ? Rompre l'hégémonie architecturale qui depuis la fin des années 1940 ne cesse de grandir. En cela, ils aspirent à casser l'idée que l'espace de l'architecture recouvre celui de la société. Ils s'engagent à montrer qu'en aucun cas ledit espace serait intégralement contenu dans une création volumétrique. En définitive : les A.J.S. manifestent - et c'est bien d'un manifeste qu'il s'agit - pour transformer les structures de pensées architecturales en se reposant non seulement sur les acquisitions technico-formelles, mais encore sur la poussée des sciences sociales récentes afin de constituer un savoir architectural et une nouvelle situation de l'architecture dans la société ${ }^{18}$.

La marche forcée chronologique a ses limites. Cependant - et ce préambule était fondamental - considérer la seconde moitié des années 1970 comme fonctionnant à partir d'une tabula rasa intellectuelle et graphique eût été d'évidence contresensuel. De fait, en 1977, à la source de L'Ivre de pierre, J.-P. Jungmann, est un architecte théoricien engagé qui réfléchit ses pratiques, son art, son travail, son insertion dans la société à la fois comme un homme de sa génération ${ }^{19}$, l'homme d'un groupe, l'homme unique au fort capital charismatique aussi dont les autres et luimême disent qu'il est "doué» $(\text { sic })^{20}$.

mars 1969 ; "Utopie ne s'écrit pas au futur ", avril 1969 ; "Le ludique et le politique ", "Critique de l'idéologie urbaine ", "Critique du concept de participation ", "De la participation des putains Nature et Culture aux agissements du maquereau Capitaliste pour perpétuer la servitude des femmes ", mai 1969 ; "Contribution à la critique radicale ", "La critique n'est pas une passion de la tête mais la tête de la passion ", "La pratique sociale de la technique " "L'argent de l'urbanisme ", décembre 1969

16. Philippe Dufieux, Sculpteurs et architectes à Lyon (1910-1960) de Tony Garnier à Louis Bertola [préface de Jean-Michel Leniaud], Lyon, Éditions Mémoire active, 2007.

17. Nicolas Poirier (dir.), "Jean Baudrillard. L'expérience de la singularité », colloque tenu à l'Université Paris Ouest-Nanterre La défense, 26-28 novembre 2014.

18. Groupe Aérolande, «Un élément oublié de l’architecture : notes sur la poésie perdue, la présence du tissu et l'usage qu'il en est fait aujourd'hui dans l'Architecture ", Revue Techniques et Architectures, 1968, no 304, p. 79-84.

19. Caroline Maniaque, "Exposition Jean-Paul Jungmann et les utopies des années 60 ", exposition de la Bibliothèque des Arts décoratifs en 2010, Archiscopie, no 99, décembre 2010, p. 20-22.

20. Entretien de J.-P. Jungmann avec Agnès Callu le 15 janvier 2015. 


\section{L'Ivre de pierres}

Avec L'Ivre de pierres - soit quatre tomes publiés en 1977, 1978, 1980 et $1984^{21}$ en grand format proches des revues de bandes dessinées - (et dans ce cadre, Aérolande quitte sa fonction de structure « commerciale " pour devenir une maison d'édition), J.-P. Jungmann crée la rupture. De fait, il suggère, par-delà l'utopie, un dessin poétique et Pierre Joly, dans une livraison de L'CEil au printemps 1978, de saluer un "dessin montrant les chemins de l'invention" (sic). On y arpente les "Sentiers de la création " inventés chez Skira par le philosophe Gaëtan Picon ${ }^{22}$, découvrant "des norias remuant les eaux mortes de canaux emmurés, des biplans aux ailes entretoisées survolant, vaguement anachroniques, des cités industrielles qu'aurait pu tracer le crayon de Tony Garnier ${ }^{23}$ ".

D'abord, il apparaît fondamental de désigner les enjeux et les catégories d'une telle initiative artistique. Les enjeux ? Un esprit chagrin les inscrirait du seul côté de la crise économique qui multiplie le chômage en même temps qu'il décroît les commandes. Pourtant investir le dessin - et lui seul - pour les architectes ne peut seulement s'analyser comme le moyen d'échapper aux circuits habituels de l'architecture (financements versus chantiers). Il ne doit non plus se comprendre à la seule lumière d'un désir de reconnaissance par les architectes d'être considérés, vus, reconnus, compris (enfin) comme des artistes produisant des créations, des livres d'artistes, singulièrement, au contenu et à la valeur esthétique (marchande aussi quand un dessin de Gaetano Pesce se vend quelque 80000 francs d'alors) ${ }^{24}$.

Ces raisons évidentes - et il est impossible d'en faire l'économie dans un dispositif démonstratif - sont cependant (et c'est presque tautologique de le rappeler) contingentes. Pourquoi ? Parce que le dessin se situe dans "l'architecture de papier» (sic). Au coude des années 1970, correspondant, sur une échelle extra-hexagonale, à un renouvellement, presque un retournement épistémologique du dessin d'architecture, il est possible de définir trois catégories de dessins : le dessin en tant qu'instrument d'élaboration du projet; le dessin comme représentation finale du projet, c'est-à-dire comme "rendu »; le dessin au titre d'objet architectural lui-même.

Ce dernier courant est celui qui intéresse, questionne, soulève J.-P. Jungmann quand il y est question d'ouverture. On le comprend d'instinct : le dessin offre des champs d'architecture beaucoup plus vastes que la réalité construite ; il permet une réflexion hors limites sur les espaces, les formes, les géométries, les matières, les couleurs. Il occupe une fonction critique à l'égard de la production d'alors; il reconfigure les rapports habituels entre forme et fonction recherchant de nouveaux modes de représentation portés par des techniques graphiques renouvelées. Il com-

21. Jean-Paul Jungmann, "L'Ivre de pierres ", L'Ivre de pierres, AJS Production, 1977.

22. Agnès Callu, Gaëtan Picon (1915-1976) : Esthétique et Culture [préface de Jean-François Sirinelli et postface d'Yves Bonnefoy], Paris, Éditions Honoré Champion, 2011.

23. Pierre Joly, L'Art, l'architecture et le mouvement moderne, Paris, Édition de La Villette, 1994.

24. François Barré (dir.), Gaetano Pesce: le temps des questions, Paris, Centre Pompidou, 1996. 
pose, au réel, une part de la recherche architecturale au même titre que la recherche théorique ou historique.

De surcroît, et pour rester dans un processus de catégorisation, il faut séparer, dans la pratique du dessin, deux tendances : d'un côté, la théorique pour laquelle le dessin d'architecture sert à illustrer des positions sur l'habitation, la ville ou l'histoire (et de ce côté, on pense à Aldo Rossi et ses collages urbains ${ }^{25}$, John Hejduk et ses abstractions géométriques ${ }^{26}$, aux frères Krier encore ${ }^{27}$. Il est possible d'y inclure le dessin polémique, celui qui, dans un concours, refuse d'assumer tels contrainte ou programme); de l'autre, se déploie la "tendanza" romanesque qui propose une vision romantique du projet, un idéal graphé où le texte occupe une place au sein du dessin lui-même, dans lequel le programme se voit défini sur des bases littéraires et poétiques, ou la "progettazione" y est décrite, de façon exhaustive, parfois de sa construction jusqu'à sa ruine. Et J.-P. Jungmann, bien sûr, Aubert, mais aussi Gaetano Pesce hanté par les lieux souterrains, Emilio Ambasz, Raimund Abraham, Anne et Patrick Poirrier avec leurs villes calcinées, s'y retrouvent ${ }^{28}$.

Stinco décrit le travail de J.-P. Jungmann, célébrant chez son ami, trois trends identitaires : la puissance du gratuit, l'intensité du désir, la conjonction positive entre dessin et écriture ${ }^{29}$ :

"Les projets de J.-P. Jungmann et Aubert seront peut-être taxés de mauvais goût. Leur forme est loin des élégances abstraites, des axonométries à l'atmosphère raréfiée [...]. Ils veulent un dessin qui puisse jouer un rôle d'exploration sensible de l'imaginaire, instrument plus proche de l'intuition, révélateur d'images risquées et même inavouables par leur naïveté et qui rappelle par la discipline qu'elle impose qu'il est la seule pratique "manuelle" de l'architecte. Il s'agit surtout de chercher, dans la narration architecturale une verve perdue, la joie d'inventer des formes arrachées aussi à la fatalité du goût, du quotidien, du prévu [...]. Si tous ces projets ont quelque chose à faire avec l'utopie, c'est qu'ils sont le fruit du désir et non de la résignation et de la soumission à la commande; ils ont plus à voir avec la séduction qu'ils ne sont tentés par la vérité $[. .$.$] . Lequeu { }^{30}$ disait "écrire ses architectures". Les textes apportent dans tout ce travail, fait du "matériel" hybride qui est celui des architectes quand le bâti ne vient pas sanctionner définitivement leurs formes, le calme de la régularité merveilleuse de la ligne écrite, la beauté

25. Aldo Rossi, Dessins, 1990-1997, Paris, Actes Sud, 1999.

26. Sanctuaries, the last works of John Hejduk: selections from the John Hejduk archive at the Canadian centre for architecture, Montreal \& the Menil collection, Houston, New York, Whitney Museum of American Art, 2003.

27. Rob Krier, Town spaces: contemporary interpretations in traditional urbanism, Basel, Birkhäuser, 2003.

28. Fulvio Irace, Emilio Ambasz: Una technological arcadia, Milan, Skira, 2004 ; Brigitte Groihofer, Raimund Abraham,Vienne, New York, Springer, 1996 ; Anne et Patrick Poirier, Dans les nervures du temps: entretiens avec Françoise Jaunin, Lausanne, La Bibliothèque des Arts, 2013.

29. Antoine Stinco, Une Éducation architecturale: Tunis, Paris, Rome, Paris, Sens et Tonka, 2014.

30. Philippe Duboÿ, Jean-Jacques Lequeu : une énigme, Paris, Hazan, 1987.

Livraisons J'bistoire de l'architecture $n^{\circ} 30$ 
de la typographie sur la feuille blanche et toute la richesse expressive du texte dans la simplicité formidable d'une feuille.»

En synthèse, et " grand angle ", dans l'exaltation d'une utopie celle qui salue l'eutopia: lieu agréable plus que l'outopia : sans lieu, le dessin chez Jungmann explique moins un projet qu'une attitude générique, globale vis-à-vis de l'urbain. Selon l'architecte, écrire un projet s'apparente à de la recherche fondamentale. "Le projet d'architecture, est une activité intellectuelle et théorique, un instrument de réflexion et de recherche, un regard singulier à l'écoute de la rumeur de la cité, immanent à sa modernité et son hasard" (sic). Le projet est premier car il :

"Dit la ville chaotique et réfractaire à sa propre théorie mais dit aussi son utopie et son "autre côté", non pas l'utopie hors du territoire ou du temps, mais celle concrète, la pensée du présent qui s'arrange avec le réel en le fondant dans une harmonie personnelle, jardin privé d'un quotidien plus exaltant, vision d'un mieux, rêverie primordiale origine de toute production poétique, prélude de l'art et de l'imagerie architecturale ${ }^{31}$."

À écouter J.-P. Jungmann, la conception de la ville utopique est donc une pensée en trois dimensions qui considère la ville comme une énorme machine - machinerie et son milieu physique comme déterminant pour l'ensemble de la vie urbaine. C'est dit : architecture et urbanisme constituent un potentiel de rupture et d'innovation dans un environnement de progrès.

\section{La colline de Chaillot}

Alors, critique et révolutionnaire pacifiste, J.-P. Jungmann, dessine et écrit/écrit et dessine dans des manifestes graphiques, jamais cyniques mais toujours poétiques quand, adossés à un dossier critique faits de photographies, de cartes postales, d'imageries mentales surtout pour certaines empruntées à Cendrars, il pense, fantasme, rêve la colline de Chaillot en 1977. Là-bas, entre dessin d'architecture et bande dessinée d'anticipation, la poésie du cosmos sensible, le premier soleil, le préau du matin, les ombres du Levant, le socle de l'été, la vallée des jeux d'eau et les portiques ruisselants du Midi, baignent une forêt de murs, d'élévations graphiques, de falaises de verre, de pentes crépusculaires de l'automne ou de vitres frileuses du soir.

Et la "Colline ", J.-P. Jungmann ne la dessine pas "en live " mais en rêve, une rêverie vagabonde effectuée depuis son atelier inscrit au cœur de la maison, de la vie socialisée. Les traits y sont libres, vifs. Rouges, verts pour certains mais selon des choix chromatiques non esthétisants, guidés par la seule recherche typographique tant l'homme n'oublie pas le réel de son goût pour l'imprimerie et les techniques (ill. 1, ill. 2, ill. 3). Comprendre les enjeux d'un tel projet (soit trente-huit planches

31. Jean-Paul Jungmann, "Architecture et utopie ou le rêve de l'architecte ", Séminaire de l'Institut parisien de recherche Architecture, urbanisme et sociétés (IPRAUS), "Architecture et Utopie ", Jean-Louis Violeau (dir.), Paris, École d'architecture de Paris-Belleville, 4 avril 2001. 


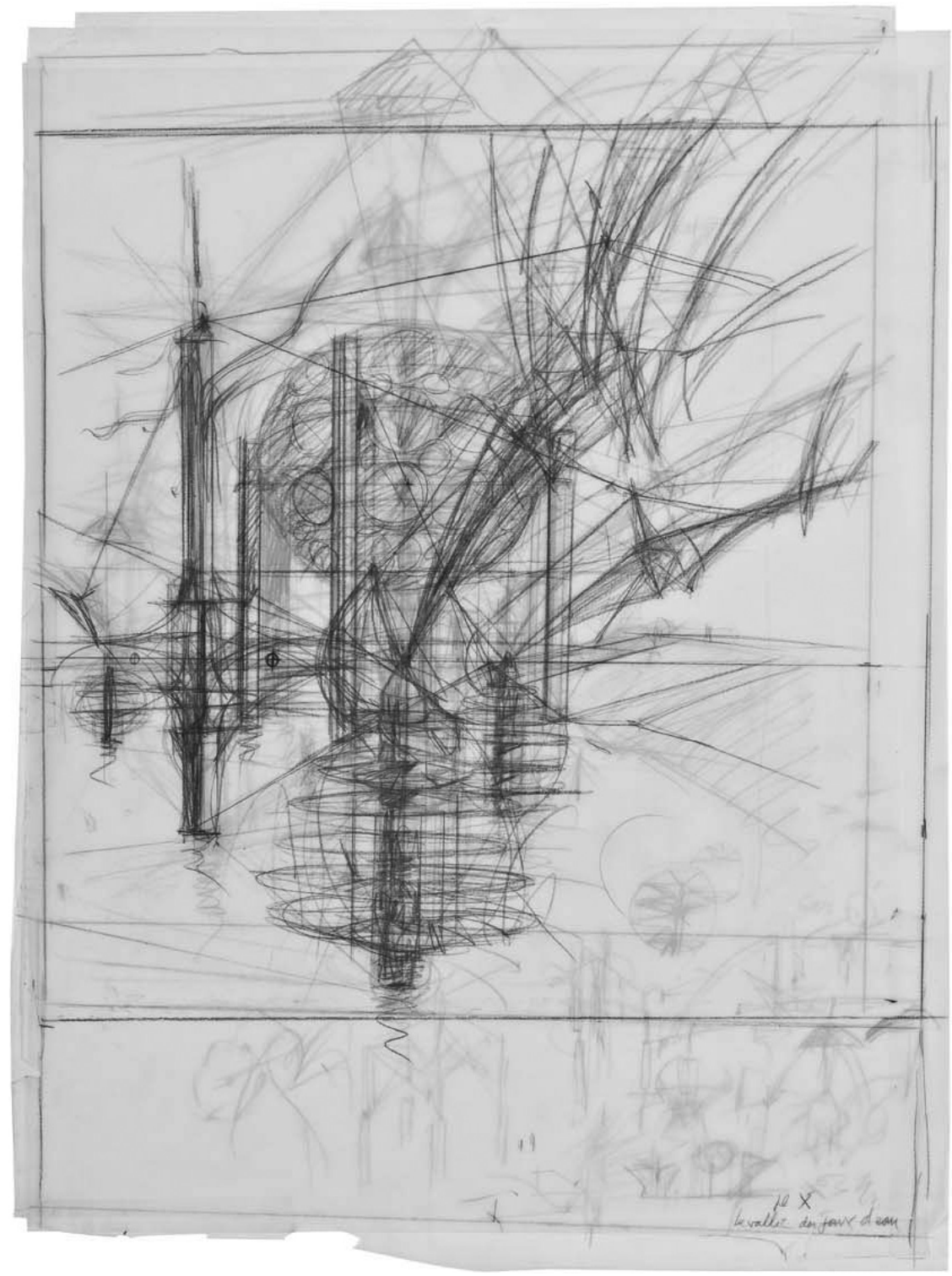

Ill. 1 : Jean-Paul Jungmann, Récit autour d'une ruine future sur la colline de Chaillot ou Le Bel Aujourd'hui, La vallée des jeux d'eau (pl. X), 1977-1978. (C) Musée des Arts décoratifs, Paris, Inv. 2010.85.3, don Jean-Paul Jungmann, 2010. 


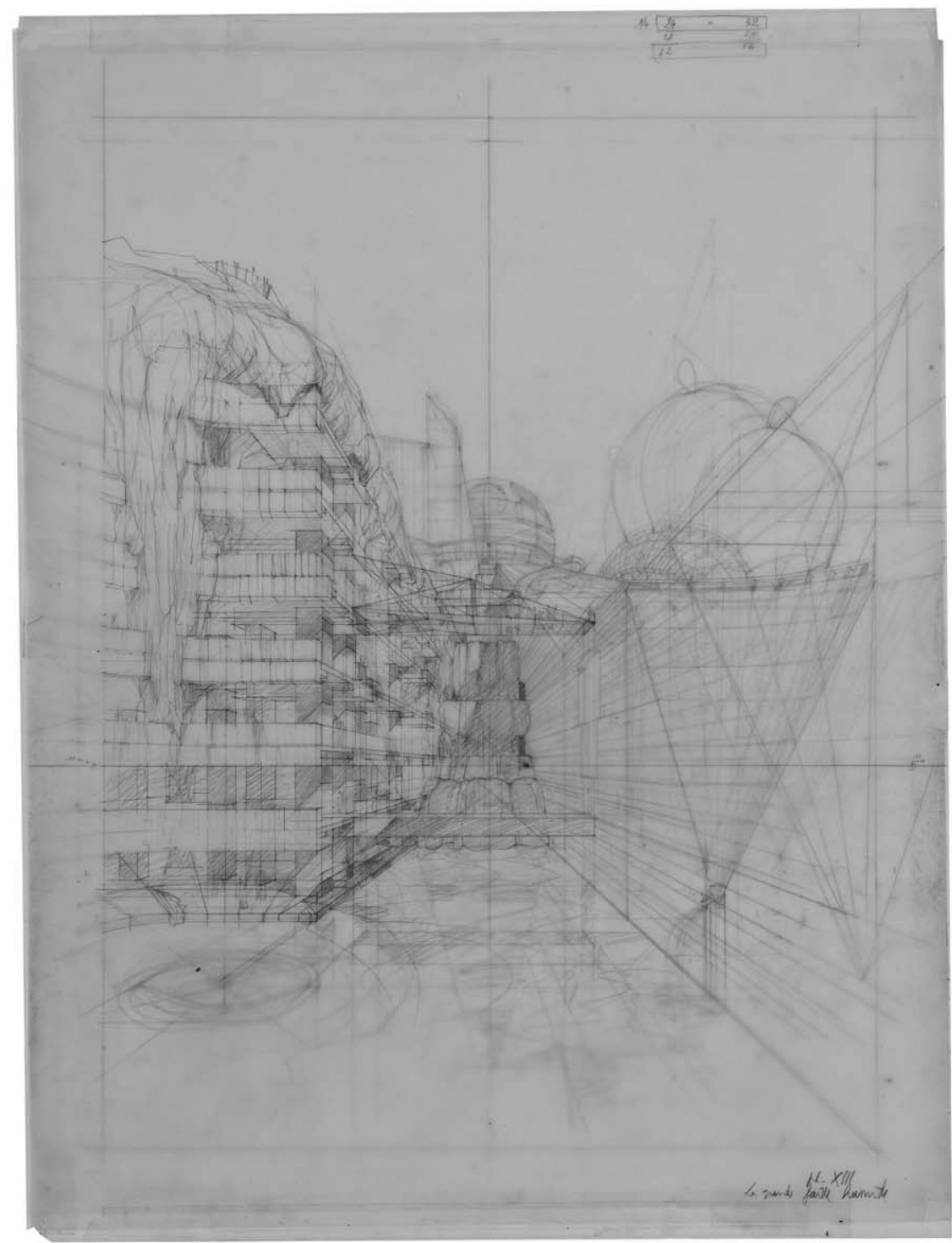

Ill. 2 : Jean-Paul Jungmann, Récit autour d'une ruine future sur la colline de Chaillot ou Le Bel Aujourd'hui, La grande faille humide et les cascades (pl. XIII), 1977-1978. (C) Musée des Arts décoratifs, Paris, Inv. 2010.85.4, don Jean-Paul Jungmann, 2010. 


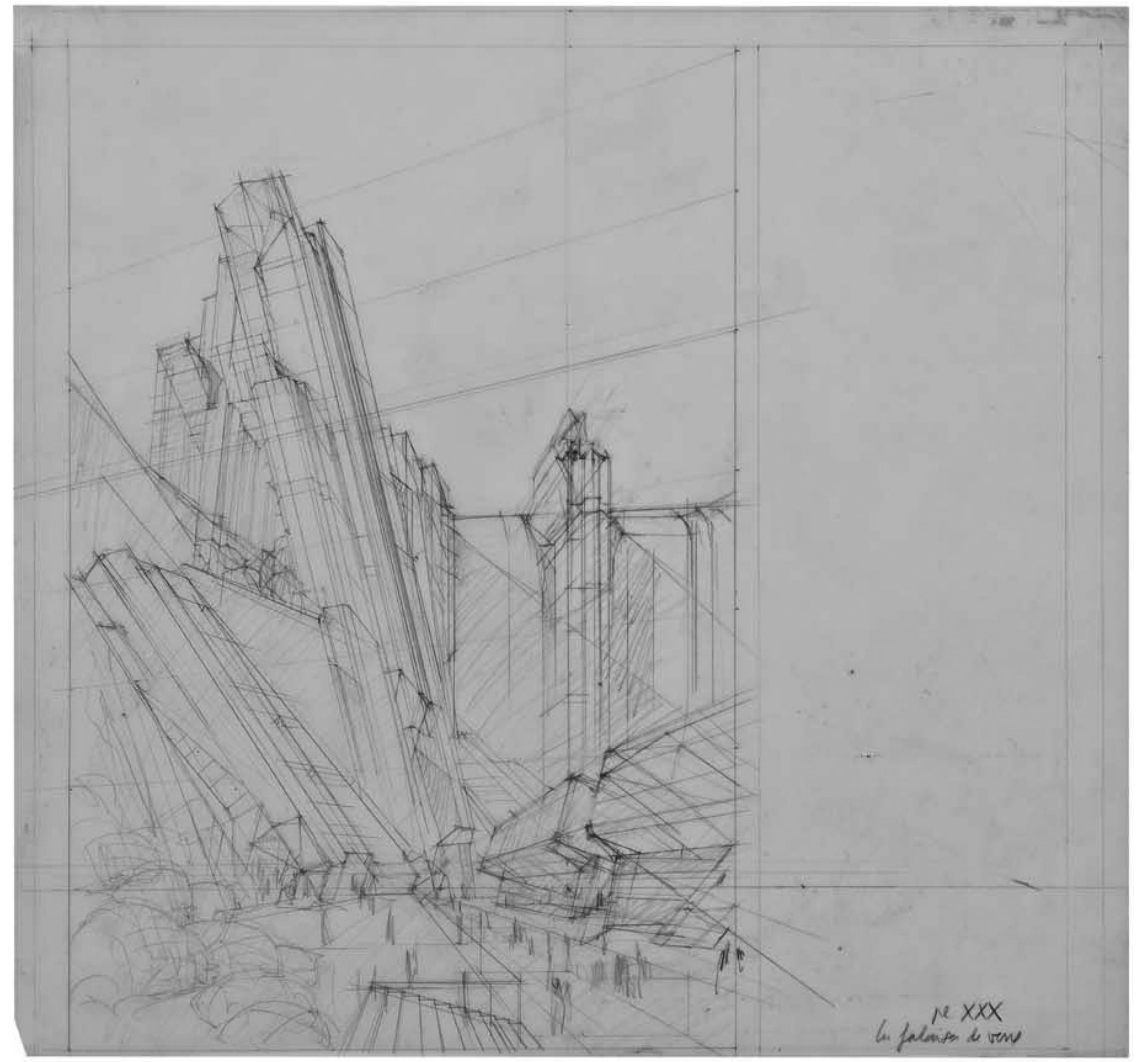

Ill. 3 : Jean-Paul Jungmann, Récit autour d'une ruine future sur la colline de Chaillot ou Le Bel Aujourd'hui, Les falaises de verre et le palais métallique de la minéralité (pl. XXX), 1977-1978. (C) Musée des Arts décoratifs, Paris, Inv. 2010.85.9, don Jean-Paul Jungmann, 2010.

de dessins de grand format au crayon rouge), oblige à avoir à l'esprit, d'abord, la notion d'utopie concrète nourrie du concept " lieu vide/lieu plein "; ensuite, de recevoir les places et rôles assignés à chacun ; enfin, de saisir, au centre, la fonction sociale et symbolique que J.-P. Jungmann assigne au dessin ${ }^{32}$.

Premièrement, à entendre J.-P. Jungmann, l'architecte utopiste doit se positionner dans un lieu (et non pas dans le non-lieu de l'utopie) ${ }^{33}$. Il lui appartient alors de voir quelles sont les potentialités du lieu en question, et plus encore, ce qui lui manque. Ce questionnement-là est fondateur car il participe soudain, par une esthétique de l'ajout, à construire une pensée, une programmation, une formali-

32. Jean-Paul Jungmann, "L'image de l'image ", Images et imaginaires d'architecture, Paris, Centre Georges Pompidou, 1984.

33. Jean-Paul Jungmann et Paul Virilio, "La cité idéale : un projet d'exposition et un thème de recherche ", novembre 1989 . 
sation, en somme, un projet d'architecture. J.-P. Jungmann est sur ce point très explicite: "C'est le manque des choses qui fait que l'on projette en avant ce que l'on aimerait bien vivre ou y vivre quand il s'agit d'un lieu ${ }^{34}$."

Deuxièmement, trop souvent, dans les projets, J.-P. Jungmann ne voit que trop courtes (décevantes aussi) les pratiques sociales et urbaines, rarement de la contextualisation ample et inventive de l'architecture. Il rêve de conjonctions de profils, c'est-à-dire que les mêmes auteurs/artistes/architectes/penseurs fassent les projets d'architecture et en écrivent le programme. La disjonction des actions, du travail et des tâches est la source première, selon lui, de la faiblesse architecturale contemporaine.

Troisièmement, J.-P. Jungmann pose comme charte que « le dessin est une projection $"(s i c)^{35}$ :

«Vous accélérez la pensée en dessinant. Quand je dessine, j’aide les autres à voir. Et puis, j’y joins ma façon d'imaginer dans l'espace. La vision dans l'espace se perd un peu. Les jeunes architectes, parce qu'ils n'apprennent à dessiner que sur des ordinateurs et presque plus à la main, ont du mal à voir en trois dimensions. Cette qualité de pouvoir imaginer dans toutes les directions, de visualiser en croquis est une qualité qu'il faut cultiver, un apprentissage par le dessin manuel $^{36}$.»

La conclusion souligne deux traits, l'un biographique, l'autre graphique. J.-P. Jungmann est signé de sa génération en sorte qu'il est infusé à l'univers esthétique et aux outils théoriques, mêlés, de Piranèse, de la revue Archigram $^{37}$ (ellemême influencée par l'univers fantastique de la bande dessinée) ${ }^{38}$, de Buckminister

34. Jean-Paul Jungmann et François Billard, «Le bal du millénaire : un événement musical et architectural, une allée musicienne de toutes les musiques festives de cette fin du $\mathrm{XX}^{\mathrm{e}}$ siècle, pour un bal de 4 km de long à Paris, capitale de la musique populaire ", juin 1997. En 1989, J.-P. Jungmann, rêve, avec Tonka, de "Tuileries révolutionnaires » : " [...] Une nouvelle vision d'un jardin historique, le temps d'une célébration; cheminements, perspectives, allées et bosquets révélés par une scénographie de fabriques, un parcours historique, symbolique et rituel, un dispositif paysagé et architectural constitué d'œuvres d'art contemporaines, éphémères pour une grande partie d'entre elles, œuvres spécialement conçues à cet effet : raconter et évoquer le mythe de 89. Le Jardin des Tuileries, transformé en exposition nationale et jardin du Bicentenaire, garderait sa fonction initiale du jardin urbain, gratuit et ouvert au public tous les jours et toute la journée, mais aussi le soir exceptionnellement où une mise en lumière spécifique ferait apparaître une autre vision, à la fois $\mathrm{du}$ jardin actuel et de ses nouvelles installations. Ce concept du jardin révolutionnaire sera celui d'un défi lancé à l'art contemporain, celui de sa possibilité d'exprimer, d'évoquer, de ritualiser les cérémonies d'une commémoration ou célébration de cette nature [...]. »

35. Jean-Paul Jungmann, "Scénographie urbaine et écriture multimédia », 2000-2001, École d'Architecture de Paris La Villette.

36. Jean-Paul Jungmann, "La cité idéale et les projets théoriques de villes : thèses provisoires sur l'attitude utopique et sa place dans la culture urbaine ", étude provisoire, mars 2000.

37. Dance with Archigram, Séoul, Space Publishing, 2005.

38. Brigitte Lemaine, "Pour une architecture fantastique ", Anne Cauquelin (dir.), thèse soutenue à Paris X, 1981. 
Fuller $^{39}$, de l'ingénieur Le Ricolais concepteur de structures légères et spatiales ${ }^{40}$ ou de Moebius ${ }^{41}$. Mais il est aussi, lui-même, un rêveur. Il faut citer Tonka ${ }^{42}$ :

"Depuis longtemps, l'art comme le monde sont constitués de deux parties. Ils sont formés de rêveurs et de pilleurs. Les pilleurs de rêves accèdent au pouvoir, méprisent les rêves que viennent d'éructer leur propre corps, se parent des rêves des autres et leurs parures séduisent rêveurs et pilleurs futurs. "

Quant à la "pensée-dessin » de J.-P. Jungmann, émanation du trinôme œil/ main/papier, elle est celle de la reconnaissance, de la croyance en une architecture, une société, un monde dont le geste graphique, dans la gratuité de son élan, la force de son talent, peut faire bouger/vaciller les lignes. Traits utopiques, graphes fantasmés, enchâssements de ductus anticipatifs, le dessin quitte la marge pour s'installer au centre. Les études de marché indiquent que L'Ivre de pierres subit le silence des médias architecturaux et enregistrent des ventes demeurées marginales. Pour autant, plusieurs générations d'architectes ont rêvé et continuent de le faire devant "l'univers dessin" de J.-P. Jungmann tant, et Bachelard le rappelle, «le rêve est à l'écart de toutes les échelles de comptage ${ }^{43}$."

Agnès CALLU

Institut d'histoire du temps présent | Centre national de la recherche scientifique

39. Buckminster Fuller, Scénario pour une autobiographie, Paris, Éditions Images modernes, 2004.

40. Le Ricolais : espace, mouvement et structures, Nantes, Musée des Beaux-Arts, 1968.

41. Moebius transe forme, Paris, Fondation Cartier pour l'art contemporain, 2010.

42. Voir, en particulier, Hubert Tonka, Fiction de la contestation aliénée : la contestation se fonde dans sa propre quotidienneté, le monde se retourne, Sens et Tonka, 2001.

43. Gaston Bachelard, La Poétique de la rêverie, Paris, PUF, 2009.

Livraisons d’bistoire de l'architecture $n^{\circ} 30$ 